\title{
Super Hydrophilic Surface Fabricated on Alumina Ceramic by Ultrafast Laser Microprocessing
}

\author{
Zhengsen Wang ${ }^{* 1}$ and Yingchun Guan *1,2,3,4 \\ ${ }^{1}$ School of Mechanical Engineering and Automation, Beihang University, 37 Xueyuan Road, Beijing 10083, China \\ ${ }^{2}$ National Engineering Laboratory of Additive Manufacturing for Large Metallic Components, Beihang University, 37 \\ Xueyuan Road, Beijing 10083, China \\ ${ }^{3}$ International Research Institute for Multidisciplinary Science, Beihang University, 37 Xueyuan Road, Beijing 100083, \\ China \\ ${ }^{4}$ Ningbo Innovation Research Institute of Beihang University, Beilun District, Zhejiang, 315800, China \\ *Corresponding author's e-mail: wzs970818@buaa.edu.cn
}

guanyingchun@buaa.edu.cn

\begin{abstract}
Super-hydrophilic alumina ceramic materials have wide application prospects in various fields and have drawn broad research interest. The failure of the hydrophilic surface is currently the main problem that limits its practical application. We have developed a simple method to directly fabricate super-hydrophilic surface on alumina ceramic by ultrafast laser micromachining. The super-hydrophilic surface fabricated on alumina ceramics by femtosecond laser can maintain the super-hydrophilic property at $0^{\circ}$ for 48 hours. The contact angle of $54.9^{\circ}$ still have hydrophilic properties after 240 hours. This is due to the increased surface roughness and the change of chemical composition on the ceramic surface treated by ultrafast laser. The groove surface with V-shaped profile enlarging the contact angle hysteresis and with capillary forces improving the spreading wetting of liquid along the grooves reduce the contact angle directly. The increase in the polar groups of surfaces and the polar component of the surface energy after laser treatment also improve the behavior of wetting and adhesion. It is noted that surface morphology plays a leading role in enhancing the wettability. This research provides guidelines for designing the surface micro-structuring based on desired geometric features and properties of alumina ceramic materials.
\end{abstract}

DOI: 10.2961/jlmn.2021.03.2005

Keywords: ultrafast laser microprocessing, laser surface texturing, super hydrophilicity, alumina ceramic, wettability

\section{Introduction}

Alumina ceramic is widely used in mechanical, electronic, chemical and aerospace engineering fields due to its excellent properties, such as high strength, high wear resistance, high hardness, thermal and chemical stability and so on. The significance of super-hydrophilic surface for the ceramic materials, especially for widely used alumina ceramic, mainly lies in self-cleaning, anti-fogging property and the strong bonding interface between the substrates and other materials [1]. Ceramic substrate with super-hydrophilic surface is useful for improving coating fabrication, achieving low friction as well as developing cell-based technologies in biomedical applications.

On basis of previous work, many methods including chemical assisted techniques, UV irradiation, polymerbased coating , plasma treatment have been successfully applied to many kinds of materials to form effective hydrophilic surface to enhance the wetting ability[2-7].These methods have many limitations in practical application, the typical disadvantages are summarized as following[3-5,710]:(a)The multistep operations and procedures are complex and slow;(b)The substrates need to expose to liquid organic or inorganic solutions;(c) Some chemical methods may cause pollution.
Laser microprocessing has attracted extensive interest and produced various well controlled microstructures on ceramic materials due to precise, green, flexible process and high production efficiency. Clemens Kunz et al. [11] fabricate laser-induced periodic surface structures on the metal phase of $\mathrm{Al}_{2} \mathrm{O} 3-\mathrm{nZrO} 2-\mathrm{Nb}$ (78.3-1.7-20 vol. \%) ceramic matrix composites. The water contact angle of composite surfaces is successfully reduced from $68.4^{\circ}$ for untreated samples to $40.9^{\circ}$ for structured samples. D. Triantafyllidis et al. [12] investigate the effect of laser surface treatment on the fluid contact angles of $\mathrm{Al}_{2} \mathrm{O}_{3}$-based refractory ceramics. The untreated surfaces demonstrate considerable non-uniformity in wetting compared with the treated surfaces and the wettability is enhanced (The contact angle reduces from $41.4^{\circ}$ to $26.9^{\circ}$ ) due to the increasing surface roughness which increases with laser power density. D.Triantafyllidis [13] enhanced the wetting ability of ceramic materials by laser treatment and the contact angle close to $25^{\circ}$.Zhou et al. [14] have carried out work on laser coating of aluminium alloys with ceramic materials silicon oxide, alumina, etc., reported that generated oxide layers often promote metal/oxide wetting. Kappel [15] has shown that the texturing of ceramics with an excimer laser can improve the adhesion strength by up to $20 \%$. However, with the passage of time, the wettabil- 
ity is prone to failure, and the failure of the hydrophilic surface is currently the main problem that limits its practical application.

In this work, we present a facile one-step methodology for fabricating super hydrophilic surface at alumina ceramic substrate by ultrafast laser.The super hydrophilic surface transformed to stable hydrophilic surface finally. Different microgroove structures are fabricated on the surface by picosecond laser or femtosecond laser in the air or Ar atmosphere to research the effects of laser parameters and processing conditions on the surface roughness. Furthermore, we analyze the effect of the change of surface roughness factor and chemical composition on the surface wettability. This research provides guidelines for designing the surface micro-structuring based on desired geometric features and properties of alumina ceramic materials.

\section{Material and methods}

\subsection{Preparation of samples}

The materials studied were commercial alumina-based ceramic. The alumina grains consisted of $0.5 \%$ magnesia. The dimension of alumina-based ceramic specimens was 10 $\mathrm{mm}$ by $10 \mathrm{~mm}$ by $3 \mathrm{~mm}$. All specimens were ground with progressively finer SiC paper (180, 400, 800, 1200, 2400 and 4000 grit), and cleaned with alcohol to reduce variation of laser beam absorption by the contaminants.

\subsection{Laser processing}

Laser-induced periodic surface structure (LIPSS) induced on the specimens laser-melted surface was carried out with Light Conversion pulsed Yb:KGW solid-state femtosecond or picosecond laser. The laser processing parameters are shown in Table 1 . The spot size of laser beam is 35 $\mu \mathrm{m}$. We chose the periodic parallel groove structure as the spatial distribution.The irradiated area was $6 \times 6 \mathrm{~mm}^{2}$ in square. Before turning on the laser, place the specimens on a processing platform under air environment or Argon gas protection environment at ambient temperature and pressure.

\subsection{Measurement and characterization}

Contact angle measurement was carried out in atmospheric condition at room temperature using a OCA-20 contact angle instrument (Dataphysics, Germany). The contact angle is defined as the angle between the solid surface and a tangent, drawn on the surface of liquid droplet, passing through the solid-liquid-vapor triple-point. Deionized water was used with a drop volume of 5ul. The contact angle instrument utilizes a precision camera and advanced PC technology to capture the image of the droplet and determine a tangent line for the WCA (Water Contact Angle) measurement. Each measurement was repeated 20 times and the average contact angle was used in the calculation.

After irradiation, sample was ultrasonically cleaned with ethanol. Surface micro/nano structures of irradiated areas were characterized by using a scanning electron microscopy (SEM, SU8010, Hitachi) and surface topography was employed using confocal laser scanning microscopy (VKX100, KEYENCE).

X-ray Photoelectron Spectroscopy (XPS) analysis was carried out for the surface chemistry using Thermo Escalab
250Xi instrument (power 150W, spot diameter 500um, voltage $14.8 \mathrm{KV}$, electricity 1.6A). Survey spectra and high-resolution spectra were acquired for surface composition analysis and chemical state identification, respectively. The charge correction of C1s (the contaminated carbon) was carried out using $284.8 \mathrm{eV}$. The narrow sweep and wide sweep pass energy (Ep) of constant analyzer were $20 \mathrm{eV}$ and $100 \mathrm{eV}$, respectively.

Table 1 Laser processing parameters in the experiments

\begin{tabular}{lcc}
\hline Parameters & $\begin{array}{c}\text { Femtosecond } \\
\text { laser }\end{array}$ & $\begin{array}{c}\text { Picosecond } \\
\text { laser }\end{array}$ \\
\hline Wavelength $(\mathrm{nm})$ & 1030 & 1030 \\
Repetition rate $(\mathrm{KHz})$ & 100 & 100 \\
Power $(\mathrm{W})$ & 8 & 12 \\
Scanning spacing $(\mu \mathrm{m})$ & 50 & 50 \\
Scanning speed $(\mathrm{mm} / \mathrm{s})$ & 30 & 15 \\
Scanning times & 30 & 15 \\
Spot diameter $(\mu \mathrm{m})$ & 35 & 35 \\
Fluence $\left(\mathrm{J} / \mathrm{cm}^{2}\right)$ & 8.32 & 12.47 \\
Pulse width $(\mathrm{ps})$ & 0.21 & 10 \\
\hline
\end{tabular}

\section{Results and discussion}

\subsection{Wettability analysis}

\subsubsection{Wettability analysis for smooth surface}

It is well known that the liquids or solids have the surface atoms or molecules energy which is higher than the interior energy so that the surface tension or free surface energy is an important surface property. This property is characterized quantitatively by the surface tension or free surface energy $\gamma$. The $\gamma$ of a unit area of the surface at constant volume and temperature conditions is equal to work. The units of $\gamma$ is $\mathrm{J} / \mathrm{m}^{2}$ or $\mathrm{N} / \mathrm{m}$ and $\gamma$ can be interpreted either as energy of per unit surface area or as tension force of per unit length of a line at the surface.

When a solid is in contact with liquid, the molecular attraction will decrease the energy of the system below the sum of the two separated surfaces. This may be interpreted by the Dupre equation

$\mathrm{w}_{\mathrm{SL}}=\boldsymbol{\gamma}_{\mathrm{SA}}+\boldsymbol{\gamma}_{\mathrm{LA}}-\boldsymbol{\gamma}_{\mathrm{SL}}$ where $\boldsymbol{W}_{\mathrm{SL}}$ is the adhesion work of per unit area between two surfaces, $\boldsymbol{\gamma}_{\mathbf{S A}}, \boldsymbol{\gamma}_{\mathbf{S L}}$ and $\boldsymbol{\gamma}_{\mathbf{L A}}$ are the surface energies and surface tensions of solid against air ,solid against liquid, and liquid against air, respectively[15, 16].

When we place a droplet of liquid on a smooth solid surface, the droplet and solid surfaces could come together under equilibrium at a characteristic angle. The characteristic angle is called the static contact angle $\theta 0$.The static contact angle $\theta 0$ can be determined by the conditions of the minimized system energy[15, 17].The minimized system energy is interpreted by the equation

$\mathbf{E}_{\mathbf{T}}=\boldsymbol{\gamma}_{\mathbf{L A}}\left(\mathbf{A}_{\mathbf{L A}}+\mathbf{A}_{\mathbf{S L}}\right)-\mathbf{W}_{\mathbf{S L}} \mathbf{A}_{\mathbf{S L}}$ where ET is the total system energy, ALA and Ast are the contact areas of the liquid with the solid and air, respectively. It is assumed that the gravitational potential energy can be neglected at the condition of the droplet is small enough so that 
the total system energy ET is 0 under equilibrium, and then the equation is

$$
\boldsymbol{\gamma}_{\mathrm{LA}}\left(\mathbf{d A _ { \mathrm { LA } }}+\mathbf{d A _ { \mathrm { SL } }}\right)-\mathbf{W}_{\mathrm{SL}} \mathbf{d A _ { \mathrm { SL } }}=\mathbf{0}
$$

For a constant volume droplet, it is easy to show by geometrical considerations, that

$\frac{\mathrm{dA}_{\mathrm{LA}}}{\mathrm{dA} \mathrm{A}_{\mathrm{SL}}}=\cos \theta_{0}$

Combining Eqs.1,3 and 4, the contact angle equation is obtained

$\cos \theta_{0}=\frac{\gamma_{L A}-\gamma_{S L}}{\gamma_{L A}}$

This Eq.5 is called Young's equation about the contact angle.Eq. 5 provides with the static contact angle value at the condition of given surface tensions.

\subsubsection{Wettability analysis for rough surface}

The Young's equation (Eq.5) is valid only in the case of a smooth solid surface. If the surface is rough, the roughness effects the contact angle in following ways: the increased contact area (Ass) and the sharp edges. The roughness distribution will not affect the contact angle when the size of the droplet is smaller than the typical size of roughness details. If the size of the droplet is larger than the typical size of roughness details of a rough solid surface, the effective interfacial tension may be a complex function of the surface roughness on the basis of the Young's equation. A droplet on a rough surface Eq.4 should be modified as

$\cos \theta=\frac{d A_{L A}}{d A_{F}}=\frac{A_{S L}}{A_{F}} \frac{d A_{L A}}{d A_{S L}}=R_{f} \cos \theta_{0}$

where $\theta$ is the contact angle of a droplet on a rough surface, $\mathrm{AF}_{\mathrm{F}}$ is the projection of the solid-liquid area (AsL) on the horizontal plane or a flat of solid-liquid contact area. $\mathrm{Rf}$ is a roughness factor defined as

$\mathbf{R}_{\mathbf{f}}=\frac{\mathbf{A}_{\mathrm{SL}}}{\mathbf{A}_{\mathbf{F}}}$

The above content is Wenzel equation[18].According to Eq.6, if the liquid wets a smooth solid surface, i.e., $\cos \theta 0$ $>0$, it will also wet the rough surface with a contact angle of $\theta<\theta 0$, because of $A_{s L} / A_{F}>1$. Furthermore, the contact angle on the rough surface is larger than it on the smooth surface $(\theta>\theta 0)$ for non-wetting liquids, i.e., $\cos \theta 0<0$.

Eustathopoulos noted that the Eq. 6 is valid only for moderate values of $\mathrm{Rf}$, when $-1<\mathrm{Rf} \cos \theta_{0}<1$. For high roughness, a wetting liquid will be completely absorbed by the rough surface cavities[19]. Eustathopoulos et al. by droplets spreading along a solid surface with grooves pointed out that a sharp edge can pin the line of contact of the solid, liquid and air, which is also known as the triple line, at a position far from stable equilibrium with the contact angles different from $\theta 0$. At the sharp edge point, the contact angle maybe have any value corresponding to the contact value from the horizontal to inclined surfaces because it is not defined. For a droplet moving along the vertical direction of grooves, the triple line will be pinned at the sharp edge point until it begins to proceed to the inclined plane. The main reason that results in the pinning is change of the surface slope of the sharp edge. The dynamic maximum advancing contact angle which is also called advancing angle at the front of the droplet is interpreted by the equation

$\boldsymbol{\theta}_{\mathrm{adv}}=\theta_{0}+\alpha$

where $\alpha$ is the slope of the edge. And the dynamic minimum receding contact angle which is also called receding angle at the back of the droplet is interpreted by the equation $\theta_{\text {rec }}=\theta_{\mathbf{0}}-\alpha$

Because of the pinning, the value of the advancing angle is greater than $\theta 0$ and the value of $t$ receding contact angle is smaller than $\theta 0$.This phenomenon is well known as the contact angle hysteresis $[15,19,20]$.The hysteresis domain of the contact angle is thus defined by the equation

$\boldsymbol{\theta}_{\text {hys }}=\theta_{\text {adv }}-\theta_{\text {rec }}$

The liquid can proceed to the inclined plane easily along the surface if the contact angle hysteresis which improve the droplet move along the vertical direction of grooves is large so that the hydrophilic property of substrate materials is enhanced.

Nosonovsky and Bhushan further examined contact angle for rough surface as a function of roughness factor for various contact angles for smooth surface[21] on the basis of Wenzel equation. For the groove surface with V-shaped profile, the absolute value of slope (the tooth angle) is $\alpha$, thus, the contact angle hysteresis on the groove surface is $2 \alpha$. According to Eq.6 and Eq.7, the roughness factor is calculated as

$\mathbf{R}_{\mathrm{f}}=\frac{1}{\cos \alpha}$

The contact angle is given as

$\cos \theta=\frac{\cos \theta_{0}}{\cos \alpha}$

The $\mathrm{Rf}$ will increase as the increasing of the tooth angle. Roughness factor by using Eq.11 to calculate of alumina ceramic processing in different conditions is shown in Table 2. According to the Table 2\&Table 3, the samples treated by femtosecond laser have the longer duration of wetting ability than those treated by picosecond laser due to have larger Rf. This is in agreement with Drelich and Chibowski's finding that roughness enhances the spread of liquid[22].Rafiee et al. pointed out that the roughness amplifies the inherent wettability of the substrate material[23].This means that if a substrate material is hydrophilic, then the Wenzel model predicts that improving surface roughness of such material would display super-hydrophilic response [24-26].The groove surface with $\mathrm{V}$-shaped profile lead to pinning of the triple line because of the sharp edges so that the liquid can proceed to the inclined plane easily and move along the vertical direction of grooves. In addition to this, the grooves with V-shaped profile only provide the roughness in the vertical direction of grooves which can improve the spreading wetting of liquid along the grooves as open capillaries[21],whose capillary forces dominate wicking of liquid into the substrate materials[22].

\subsection{WCA measurement}

We measured static contact angle by placing water droplet on the original surface and laser-textured areas respectively to compare the effect of laser processing about surface wetting. As shown in Fig.1(a), the initial alumina ceramic surface exhibits hydrophobicity with the contact angle of $115^{\circ}$. After femtosecond or picosecond laser treatment, the droplet spreads rapidly with the contact angle of 0 , which indicates that the microgroove surfaces exhibit the super hydrophilic property.

Because of the laser processing treatment, the increasing of roughness and the polar functional groups contents of 
the ceramic material surface resulted in the extreme superhydrophilicity. The work of adhesion may be interpreted by the Young-Dupre equation

$\mathrm{W}_{\mathrm{ad}}=\gamma_{\mathrm{lv}}(1+\cos \theta)$

where $\gamma_{l v}$ is the liquid-vapor interfacial energy and $\theta$ is the contact angle[14].According to the Eq.13,the adhesion energy of water to the substrate is increased as the contact angle decreasing. Table 2 summarized the evolution of WCA of samples as time went on. As shown in Table 2, after exposure in ambient air for $24 \mathrm{~h}, 48 \mathrm{~h}, 72 \mathrm{~h}, 96 \mathrm{~h}, 168 \mathrm{~h}$ and $240 \mathrm{~h}$, the contact angles of the laser textured surfaces increase and then gradually stabilize over time. The surface fabricated by femtosecond laser treatment is able to keep super hydrophilic after 48 hours, and the surface fabricated by picosecond laser treatment is able to keep super hydrophilic after 24 hours. As the storage time increases, the contact angle becomes larger and the super hydrophilic property disappears. After 240 hours, the super hydrophilic surface transformed to stable hydrophilic surface. The contact angles $\left(100^{\circ}\right.$ and $102^{\circ}$ ) of the laser textured surfaces fabricated by picosecond laser in the air and Ar atmosphere are larger than the critical angle $\left(90^{\circ}\right)$ and the surfaces become hydrophobic. In contrast, the laser textured surfaces fabricated by femtosecond laser remain hydrophilic with the contact angle of $55^{\circ}$ in the air atmosphere and $68^{\circ}$ in the Ar atmosphere. It is worth noting that laser textured surfaces fabricated in the air atmosphere provide better hydrophilic performance compared with the Ar atmosphere.

Table 2 The contact angle measurement results

\begin{tabular}{lcccccc}
\hline $\begin{array}{c}\text { Processing } \\
\text { conditions }\end{array}$ & $24 \mathrm{~h}$ & $48 \mathrm{~h}$ & $96 \mathrm{~h}$ & $168 \mathrm{~h}$ & $240 \mathrm{~h}$ & $360 \mathrm{~h}$ \\
\hline $\begin{array}{c}\text { femtosecond } \\
\text { in air }\end{array}$ & 0 & 0 & 12 & 24 & 55 & 56 \\
$\begin{array}{c}\text { picosecond } \\
\text { in air }\end{array}$ & 0 & 15 & 28 & 46 & 100 & 100 \\
$\begin{array}{c}\text { femtosecond } \\
\text { in Ar }\end{array}$ & 0 & 0 & 16 & 30 & 68 & 70 \\
$\begin{array}{c}\text { picosecond } \\
\text { in Ar }\end{array}$ & 0 & 24 & 45 & 54 & 102 & 103 \\
\hline
\end{tabular}

\subsection{Surface characterization}

Fig. 1 shows the SEM images and confocal images of the cross-sectional and microstructures of laser melted alumina-based ceramic. The original alumina-based ceramic contained flat surface with many micro/nanometer pores distributed nonuniformly. After laser modification, the microstructure of the alumina-based ceramic was changed significantly. The laser-textured surfaces contained parallel grooves arranged uniformly as well as submicron features along the grooves. The laser melting produced a homogeneous and continuous modified layer. Compare to microstructure of the grooves of the specimens in Fig.1, the difference is related to the different processing environment (air or $\mathrm{Ar}$ ) and laser processing parameters such as pulse duration (femtosecond or picosecond), laser power, scanning times and so on.
It should be noted that compared to the specimens treated by picosecond laser with the roughness factor of 2.6 in the Ar atmosphere and the roughness factor of 2.3 in the air atmosphere, the specimens treated by femtosecond laser have more smooth surface, deeper groove valleys and sharper groove ridges with the roughness factor of 3.5 in the Ar atmosphere and the roughness factor of 3.1 in the air atmosphere. Especially the specimens treated by femtosecond laser in air environment have the most smooth surfaces and deepest groove valleys. Table 3 shows the microgroove width, the microgroove depth and the roughness factor of laser textured surfaces of alumina ceramic under different processing conditions. Compared with the femtosecond laser treatment, picosecond laser can provide longer interaction time with the alumina ceramic substrates. The increasing heat input can bring more heat influence to the surrounding materials, which leads to shallow microgroove structure and small surface roughness. Furthermore, the Ar atmosphere is beneficial to suppress the formation of reactants on the surface of the substrates, which can provide deeper microgroove structure and larger roughness factor.

The change of microstructure at laser-textured surfaces increases both surface area and surface roughness, which contributes to the formation of superhydrophilicity in terms of physical factors. The microstructures of laser textured surfaces rarely change with store time.

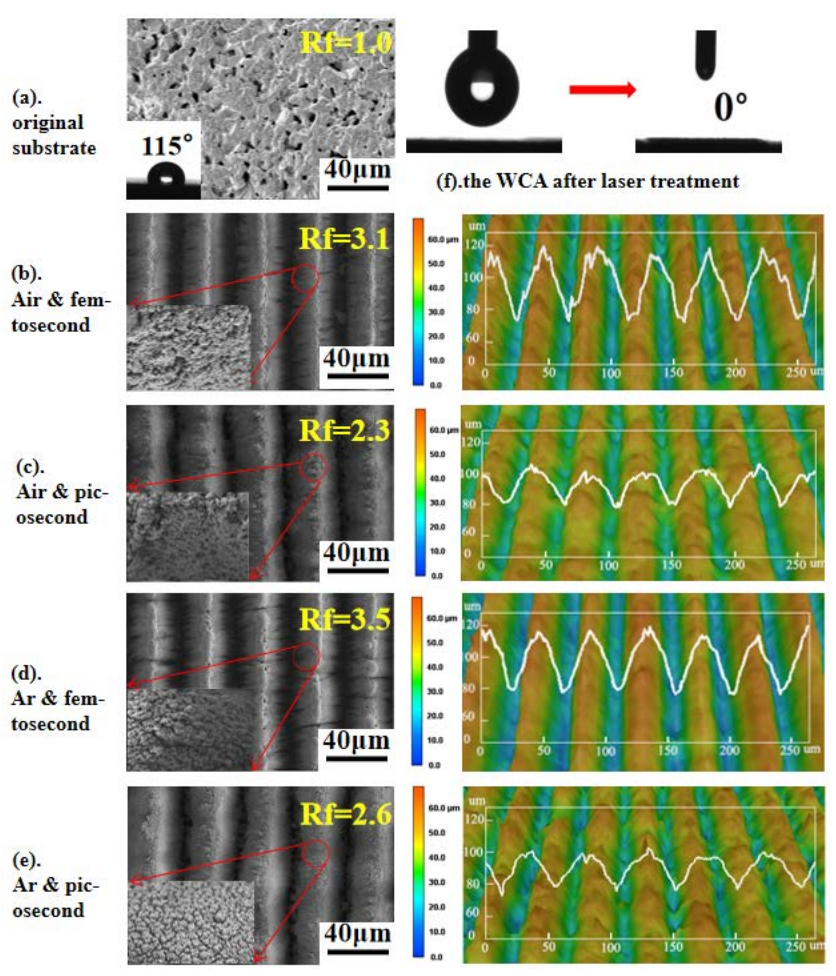

Fig. 1 Surface topography of the laser textured alumina ceramic substrates characterized by SEM and LSCM: (a) the original substrate; (b) the substrate fabricated by femtosecond laser in the $\mathrm{Ar}$ atmosphere; (c) the substrate fabricated by picosecond laser in the Ar atmosphere; (d) the substrate fabricated by femtosecond laser in the air atmosphere; (e) the substrate fabricated by picosecond laser in the air atmosphere and (f) the water contact angle (WCA) measurement after laser treatment. 
Table 3 The microgroove width, depth and roughness factor

\begin{tabular}{cccc}
\hline Processing conditions & width $(\mu \mathrm{m})$ & depth $(\mu \mathrm{m})$ & $R_{f}$ \\
\hline femtosecond in air & $49-52$ & $37-43$ & 3.1 \\
picosecond in air & $48-52$ & $21-26$ & 2.3 \\
femtosecond in Ar & $49-51$ & $38-42$ & 3.5 \\
picosecond in Ar & $48-51$ & $20-24$ & 2.6 \\
\hline
\end{tabular}

\subsection{XPS analysis}

XPS is a popular and powerful technique for the investigation of surface composition, it does provide qualitative and quantitative information on the chemical changes of materials. We used XPS to characterize the chemical composition of both fresh laser-textured surfaces and lasertextured surfaces after 240 hours. The XPS survey spectrums of both fresh laser-textured surfaces and laser-textured surfaces after 240 hours are shown in Fig. 2 and Fig.3.
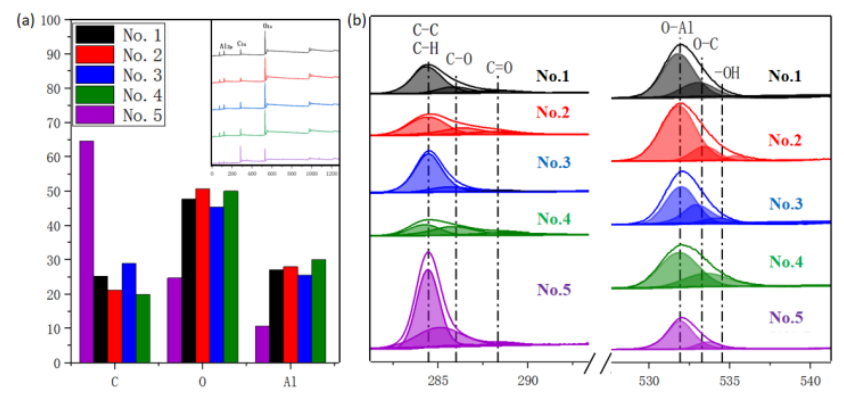

Fig. 2 XPS spectra of fresh laser textured surfaces.(a) Atomic percentage of Al, $\mathrm{C}$ and $\mathrm{O}$ elements.(b)XPS C1s spectra and O1s spectra.(No.1 is treated by femtosecond laser in air;No.2 is treated by picosecond laser in air; No.3 is treated by femtosecond laser in $\mathrm{Ar}$;No.4 is treated by picosecond laser in Ar;No.5 is original material. )
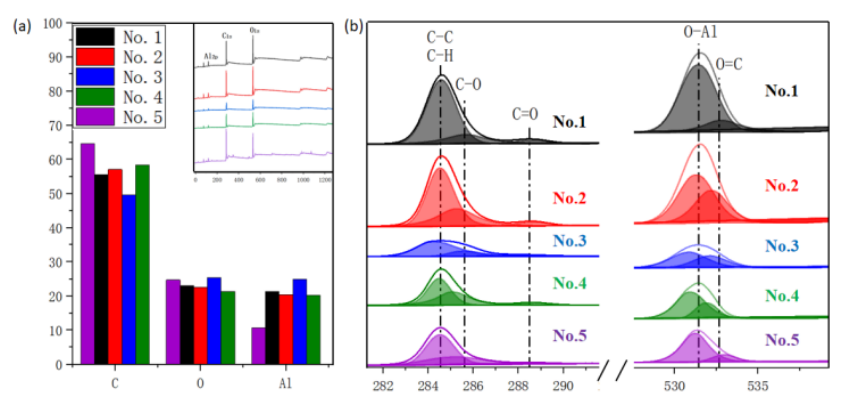

Fig. 3 XPS spectra of laser textured surfaces after 240 hours.(a) Atomic percentage of $\mathrm{Al}, \mathrm{C}$ and $\mathrm{O}$ elements.(b)XPS C1s spectra and O1s spectra.(No.1 is treated by femtosecond laser in air;No.2 is treated by picosecond laser in air; No.3 is treated by femtosecond laser in Ar;No.4 is treated by picosecond laser in Ar;No.5 is original material. )

Fig.2(a) and Fig.3(a) indicated the chemical composition almost solely consists of $\mathrm{Al}, \mathrm{C}$ and $\mathrm{O}$ elements and shows the atomic percentage of $\mathrm{Al}, \mathrm{C}, \mathrm{O}$ elements of the original surface and laser-textured surface respectively . Compared to the XPS survey spectrums of the original sur- face,the surface $\mathrm{O}$ element content of the laser-textured areas was increased prominently. The laser ablation induces the formation of reactants on the surface of the substrate, which leads to the increase of the $\mathrm{O}$ element content. The oxygen atoms of surface could form hydrogen bonds with hydrogen atoms of the water molecules to enhance wetting and adhesion ability[1, 27].However, after storage for 240 hours, the $\mathrm{O}$ element content of the laser-textured areas treated in the air atmosphere and in the Ar atmosphere respectively decreases while the $\mathrm{C}$ element content increases.

Fig.2(b) and Fig.3(b) show the C1s and O1s spectra of fresh laser textured surfaces and laser textured surfaces after 240 hours. The chemical compounds include polar functional groups and metallic oxides. The main metallic oxides were $\mathrm{Al}-\mathrm{O}$ bonds and the main polar functional groups were $\mathrm{C}-\mathrm{O}, \mathrm{C}=\mathrm{O}$. The intensity of $\mathrm{C}-\mathrm{O}$ and $\mathrm{C}=\mathrm{O}$ peaks of fresh laser textured surfaces is higher than that of laser textured surfaces after 240 hours. As we all known, water molecule is polar so that the polar functional groups enhance the wetting ability of material surface significantly.[21, 25, 28-30] What's more, the $-\mathrm{OH}$ polar functional group is observed in XPS O1s spectra, which can improve the wetting behavior significantly because of its water compatibility.

Fig. 4 shows the atomic percentage of the $-\mathrm{OH}$ bond and the $\mathrm{C}=\mathrm{O}$ bond of laser textured surfaces. The relative percentage of the $-\mathrm{OH}$ bond and the $\mathrm{C}=\mathrm{O}$ bond on fresh laser textured surfaces is higher than that on the original substrate. Because of the oxidation during the processing, the laser treatment can increase the surface oxygen content. The accumulation of oxygen element on the laser textured surfaces results in the increase of surface energy and wetting ability.

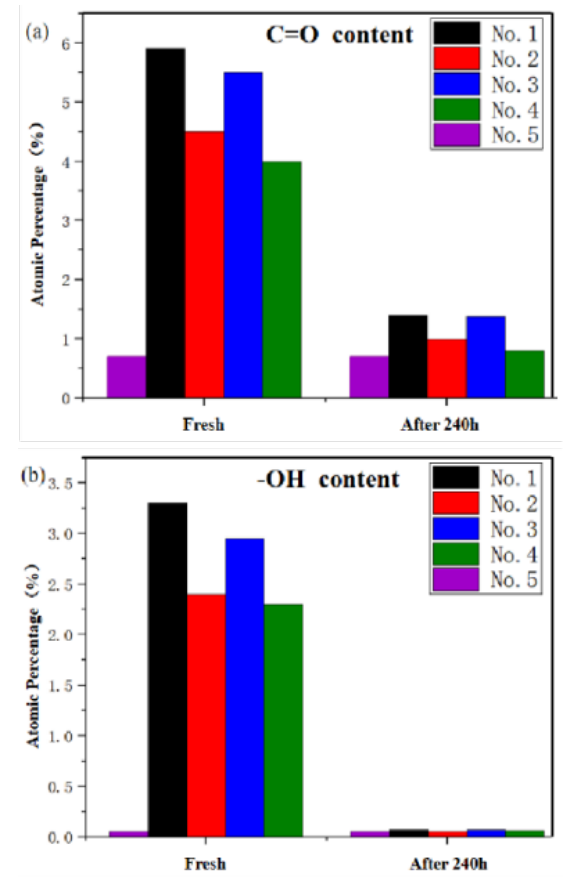

Fig. 4 Comparison of polar functional groups contents between fresh laser textured surfaces and laser textured surfaces after 240 hours. (a) Comparison of $\mathrm{C}=\mathrm{O}$ content and (b) Comparison of $-\mathrm{OH}$ content. (No.1 is treated by femtosecond laser in air; No.2 is treated by picosecond laser in air; No.3 is treated by femtosecond laser in $\mathrm{Ar}$; No.4 is treated by picosecond laser in Ar; No.5 is original material.) 
The surface energy is determined by intermolecular forces and it comprises of polar interaction and dispersive component. The work of adhesion Wad can be also expressed as the sum of different intermolecular forces that act at the interface with the dispersive component $\left(\gamma_{s v}^{d}\right.$ and $\left.\gamma_{l v}^{d}\right)$ and the polar component $\left(\gamma_{s v}^{p}\right.$ and $\left.\gamma_{l v}^{p}\right)$

$\mathrm{W}_{\mathrm{ad}}=\mathrm{W}_{\mathrm{ad}}^{\mathrm{d}}+\mathrm{W}_{\mathrm{ad}}^{\mathrm{p}}=2\left(\gamma_{\mathrm{sv}}^{\mathrm{d}} \gamma_{\mathrm{lv}}^{\mathrm{d}}\right)^{1 / 2}+2\left(\gamma_{\mathrm{sv}}^{\mathrm{p}} \gamma_{\mathrm{lv}}^{\mathrm{p}}\right)^{1 / 2}$

where $\gamma_{s v}$ and $\gamma_{l v}$ are the solid-vapor and liquid-vapor interfacial energy, respectively. J. Lawrence et al. pointed out that the action of polar forces across the interface enhances the wetting ability and water adhesion. Therefore, high content of polar functional groups will result in high polar forces so that the laser-textured areas has greater wetting ability and higher water adhesion [1, 31] .Compared with the laser textured surfaces proceeded in the Ar atmosphere, the laser textured surfaces proceeded in the air atmosphere have better hydrophilic property, because they have the higher content of polar functional groups as the $-\mathrm{OH}$ bond and the $\mathrm{C}=\mathrm{O}$ bond on the surfaces. After 240 hours, the atomic percentage of the $\mathrm{C}=\mathrm{O}$ bond of laser textured surfaces decreases and the $-\mathrm{OH}$ bond is almost disappeared. Based on the study of Shemella et al. on self-healing hydrophobic recovery, the hydrophobic recovery is suggested that caused by degradation process of surface composition such as hydrocarbon, hydration and oxide layer. The degradation process of surface composition results that there will not be sufficient exposed groups and oxidized sites to form the hydrophilic surface[27].

\section{Conclusions}

In this research, super hydrophilic surface on alumina ceramic is fabricated by ultrafast laser micromachining. Improvements in the wetting behavior of the ceramic materials after laser treatment were due to:

1)The laser treatment of the ceramic materials surfaces improves the surface roughness so that amplify the wettability of the substrate material. The groove surface with Vshaped profile enlarging the contact angle hysteresis and with capillary forces improving the spreading wetting of liquid along the grooves reduce the contact angle directly. Furthermore, the femtosecond laser treatment provides deeper microgroove structure and larger surface roughness factor than the picosecond laser treatment because of the shorter interaction time and the less heat input with the alumina ceramic substrates.

2)The laser treatment result in increasing the surface oxygen content of the ceramic materials and the oxygen atoms of surface could form hydrogen bonds with hydrogen atoms of the water molecules as further enhancing the wetting and adhesion ability.

3)The increase in the polar groups of surfaces and the polar component of the surface energy after laser treatment also improve the behavior of wetting and adhesion.

This technique has wide application prospects, such as improving coating adhesion, achieving low friction, adhesion of cells to biomaterial surfaces, etc.

\section{Acknowledgments}

This research is supported by the National Natural Science Foundation of China (51875313), National Key Research and Development Program of China (2018YFB1107700).

\section{References}

[1] J. Lawrence, L. Li, and J.T. Spencer: Appl. Surf. Sci., 138, (1999) 388.

[2] A. Passerone, F. Valenza, and M.L. Muolo: J. Mater. Sci. Lett., 47(24), (2012) 8275.

[3] T. Ishizaki, N. Saito, and O. Takai: Langmuir, 26(11), (2010) 8147.

[4] K. Chao, H. Lu, S. Yuan, D. Hong, K. Yan, and B. Liang: Chem. Eng. J., 203, (2012) 1.

[5] H.S. Lim: Adv. Mater., 20(23), (2008) 4438.

[6] J. Yuan, X. Liu, O. Akbulut, J. Hu, S.L. Suib, J. Kong, and F. Stellacci: Nat. Nanotechnol., 3(6), (2008) 332.

[7] Q. Xie, W. Wang, Z. Xie, S. Shuang, LI. Zhengcao, and Z. Zhang: Langmuir, 25(4), (2009) 1927.

[8] A.J. Lopez, B. Torres, C. Taltavull, and J. Rams: Mater. Des., 43, (2013) 144.

[9] P. Kim, TS. Wong, J. Alvarenga, and MJ. Kreder: ACS. Nano., 6(8), (2012) 6569.

[10] R. Na, L. Jianhua, Q. Jichuan, S. Yuanhua, and J. Huaidong: Small, 10(15), (2014) 3169.

[11] K. Clemens, JF. Bartolom, G. Enrico, and G. Stephan: Appl. Surf. Sci., 434, (2017) 582.

[12] D. Triantafyllidis, L. Li, and F.H. Stott: Mater. Sci. Eng., A, 290(1), (2005) 271.

[13] D. Triantafyllidis, L. Li, and F.H. Stott: J. Appl. Thin Solid Films., 453, (2004) 76.

[14] L. Marrot, A. Bourmaud, P. Bono, and C Baley: Mater. Des., 62, (2014) 47.

[15] J.N. Israelachvili: "Intermolecular and surface forces.", (Academic press, New York, 2011) p.661.

[16] B. Bhushan: "Principles and applications of tribology". (John Wiley \& Sons, New Jersey, 1999) p.635.

[17] A. Adamson: "Physical chemistry of surfaces", (Wiley, New York, 1979) p.93.

[18] R.N. Wenzel: Ind. Eng. Chem. Fundam., 28(8), (1936) 988.

[19] N. Eustathopoulos, M.G. Nicholas, and B. Drevet: Pergamon, 3, (1999) 75.

[20] F.M. Fowkes, W.A. Zisman: Adv. Chem. Phys., 2, (1964) 6.

[21] M. Nosonovsky, B. Bhushan: Microsyst. Technol., 11(7), (2005) 535.

[22] J. Drelich, E. Chibowski: Langmuir, 26(24), (2010) 18621.

[23] J. Rafiee, R. Javad, A.R. Mohammad, Y. Zhongzhen, and K. Nikhil: Adv. Mater., 22(19), (2010) 2151.

[24] R.N. Wenzel: J. Phys. Org. Chem., 53(9), (1949) 1466.

[25] T.S. Chow: J. Phys.: Condens. Matter., 10(27), (1998) 6772.

[26] P.S. Swain, R. Lipowsky: J. Appl. Langmuir, 14(23), (1998) 6772.

[27] P.T. Shemella, T. Laino, O. Fritz, and A. Curion: J. Phys. Chem. B, 116(24), (2012) 7351.

[28] D.P. Woodruff: "Modern techniques of surface science.” (Cambridge university press, London,2016) p.1. 
[29] M. Rastogi: "Surface and interfacial science: applications to engineering and technology”(Alpha Science ,London,2003) p.334.

[30] Y.C. Guan, W. Zhou, Z.L. Li, and H.Y. Zheng: Appl. Surf. Sci., 255(19), (2009) 8235.

[31] Y.C. Guan, F.F. Luo, G.C. Lim, M.H. Hong, H.Y. Zheng, and B. Qi: Mater. Des., 78, (2015) 19.

(Received: June 12, 2021, Accepted: December 21, 2021) 